\title{
A MORTALIDADE POR MALFORMAÇÃO CONGÊNITA NO MUNICÍPIO DE SÃO PAULO NO PERÍODO DE 1940 A $1964{ }^{(1)}$
}

E estudada a mortalidade por malformações congênitas no municipio de São Paulo durante um período de 25 anos, ou seja de 1940 a 1964, tendo se verificado que ela apresenta uma tendência ascendente, predomina nos menores de 1 ano em relação a tôdas as outras idades e é maior no sexo masculino. Quanto aos tipos de malformações congênitas letais mais frequientes verificou-se serem aquelas do aparelho cardiocirculatório. Discute-se as prováveis causas do aumento dos coeficientes de mortalidade por malformações congênitas.

\section{INTRODUCAO}

As malformações congênitas vem adquirindo nos últimos anos grande importância não só no que diz respeito à sua terapêutica, quer clínica ou, principalmente, cirúrgica, mas também - e talvez mais importante - devido ao estudo de sua epidemiologia, visando obter os conhecimentos necessários para se adotarem medidas preventivas.

Em países onde a mortalidade infantil é baixa, visto estarem grandemente diminuídas as doenças causadas pelos fatôres ambientais, representam as malformações congênitas importante contingente na proporção dos óbitos em menores de 1 ano. A sua prevenção, desde que conhecidas as causas faria diminuir muito mais os coeficientes de mortalidade infantil.

Muitos estudos têm sido realizados visando conhecer melhor a incidência das malformações congênitas (HIGgins ${ }^{2}$, 1965; Malpas ${ }^{3}$, 1937; McIntosh $^{4}$, 1954; ShaPiro $\left.{ }^{\circ}, 1958\right)$ e entre nós SaL-
DANHA et alii ${ }^{5}$ (1963) estudou o problema na população de São Paulo. A maioria dêsses estudos é feita retrospectivamente a partir de registros de nascimentos de hospitais e maternidades, ou então em estudos mais completos feitos prospectivamente, onde as crianças são examinadas periòdicamente, durante um certo tempo, trazendo então informaçōes mais completas, pois muitas malformações só são evidenciadas meses após o nascimento.

Tais estudos de morbidade, geralmente, permitem analisar todos os tipos de malformações, desde as mais banais (minor) até as mais graves e muitas vêzes incompatíveis com a vida (major), sendo porém de realização nem sempre fácil.

Uma maneira de se analisar o que está ocorrendo com as malformaçōes congênitas seria através das estatísticas de mortalidade. Ainda que os dados sôbre mortalidade por malformações congênitas sòmente nos dêem informaçōes sôbre

Recebido paar publicação em 14-7-1967.

(1) Da Cadeira de Estatística Aplicada à Saúde Pública da Faculdade de Higiene e Saúde Pública da USP. 
LAURENTI, R. - A mortalidade por malfo:mação congênita no Município de são Paulo no periodo de 1940 a 1964. Rev. Saúde públ., S. Paulo, 1(1):51-58, jun. 1967.

aquelas mais graves e letais, poderiam até certo ponto, refletir a incidência, senão quanto à sua magnitude, pelo menos sua tendência num determinado período.

O objetivo desta apresentação é analisar a mortalidade por malformaçóes congênitas no município de São Paulo num período de 25 anos compreendido entre 1940 e 1964, e as possíveis causas de sua variação.

\section{MORTALIDADE POR MALFORMACOES CONGENITAS EM TODAS AS IDADES}

A tendência da mortalidade por malformações congênitas, na população em geral, no município de São Paulo, de 1940 a 1964, pode ser vista na Tabela 1.

T A B EL A 1

Malformações congênitas - Mortalidade (coef. $\times 100.000$ habitantes) e mortalidade proporcional $(\%)$ em tôdas as idades - $\mathrm{Mu}$ nicipio de São Paulo, 1940-1964

\begin{tabular}{|c|c|c|}
\hline A no & Coeficiente & $\%$ \\
\hline 1940 & 7,0 & 0,50 \\
\hline 1941 & 8,0 & 0,55 \\
\hline 1942 & 3,0 & 0,50 \\
\hline 1943 & 5,0 & 0,45 \\
\hline 1944 & 6,0 & 0,40 \\
\hline 1945 & 8,0 & 0,70 \\
\hline 1946 & 8,0 & 0,75 \\
\hline 1947 & 9,0 & 0,85 \\
\hline 1948 & 9,0 & 0,80 \\
\hline 1949 & 10,0 & 1,00 \\
\hline 1950 & 9,0 & 0,90 \\
\hline 1591 & 12,0 & 1,20 \\
\hline 1952 & 11,0 & 1,25 \\
\hline 1953 & 13,0 & 1,35 \\
\hline 1954 & 14,0 & 1,50 \\
\hline 1955 & 16,0 & 1,70 \\
\hline 1956 & 18,0 & 1,85 \\
\hline 1957 & 17,0 & 1,90 \\
\hline 1958 & 18,0 & 2,15 \\
\hline 1958 & 17,0 & 2,00 \\
\hline 1960 & 17,0 & 2,00 \\
\hline 1961 & 18,0 & 2,20 \\
\hline 1962 & 18,0 & 2,00 \\
\hline 1963 & 19,0 & 2,15 \\
\hline 1964 & 15,0 & 1,85 \\
\hline
\end{tabular}

Vemos que, embora não havendo um aumento constante de ano para ano, e inclusive havendo alguns anos em que se verifica diminuição do coeficiente de mortalidade, às vêzes acentuada como de 1941 para 1942 (diminuição de $62,5 \%$ ), de uma maneira geral nos 25 anos considerados no estudo, houve uma tendência ascendente no coeficiente de mortalidade por malformações congênitas em tôdas as idades. Assim, se compararmos os coeficientes médios relativos aos triênios 1940-42, 1950-52 e 1962-64, que são respectivamente $6,0,10,6$ e $17,6 \times 100.000$ habitantes, vê-se que o coeficiente médio do triênio inicial aumentou $76,6 \%$ no triênio intermediário, e dêste para o triênio final houve um aumento de $63,2 \%$. Relacionando-se os triênios inicial e final, o aumento foi de 188,3\%.

Nota-se que de 1942 a 1956 a tendên. cia ascendente da mortalidade é mais acentuada, sendo que a partir de 1956 e até 1963 , tem-se mantido mais ou menos estacionária, sendo que no último ano estudado (1964), houve uma diminuição no coeficiente de $22 \%$ em relação ao ano anterior (1963).

A mortalidade proporcional por malformações congênitas, em tôdas as idades, e em relação a tôdas as outras causas de morte, também pode ser apreciada na Tabela 1. Vê-se que nos primeiros anos a proporção era baixa $(0,40-0,75 \%)$, e a partir de 1946-47. começa a se elevar, permanecendo desde 1957, em tôrno de $2,0 \%$, havendo portanto um aumento nos anos finais, em relação aos iniciais, de $70.75 \%$ na proporção de mortes por malformações congênitas. Podemos avaliar melhor a mortalidade proporcional em tôrno de $2,0 \%$ existentes a partir de 1957, comparando-a com a causa que teve a maior proporção de mortes, por exemplo em 1962, e que foram as neoplasias malignas correspondendo a $12,0 \%$ de todos os óbitos por tôdas as outras causas, ou seja 6 vêzes mais do que os óbitos por malformações congênitas no mesmo ano. 
LAURENTI, R. - A mortalidade por malformação congênita no Município de são Paulo no periodo de 1940 a 1964. Rev. Saúde públ., S. Paulo, 1(1):51-58, jun. 1967.

Para todos os anos estudados, predominaram os óbitos no sexo masculino, com uma média de $55,9 \%$, variando de um mínimo de $51,5 \%$ em 1945 até um máximo de $63,2 \%$ em 1940.

Também os coeficientes de mortalidade segundo sexo foram maiores para o sexo masculino em todos os anos. Em 1964, para o sexo masculino o coeficien. te foi $16,3 \times 100.000$ e para o sexo feminino, $14,5 \times 100.000$, e podemos comparar com os coeficeintes para vários países apresentados poh Higgins ${ }^{2}$ (1965) para anos próximos a 1964. Para todos os países, exceto Israel, Noruega, Irlanda do Norte e Groenlândia são maiores os coeficientes para o sexo masculino fato igualmente cbservado no Município de São Paulo.

Os coeficientes para algumas capitais brasileiras são apresentados na Tabela 2 . Seria difícil fazermos uma comparação com o observado em São Paulo, pois certamente existem variações quanto à qualidade diagnóstica, diferenças no preenchimento dos atestados de óbito e tam. bém na codificação e frequiências de outras causas de morte, principalmente moléstias infecciosas e causas mal definidas.

\section{T A B E L 2}

Mortalidade por malformações congênitas em algumas capitais brasileiras - Anos próximos a 1964 (coeficiente $\times 100.000$ habitantes)

\begin{tabular}{l|r|r|r|r}
\hline \multirow{2}{*}{ Capitais } & \multicolumn{5}{c}{ A no s } \\
\cline { 2 - 5 } & 1961 & 1962 & 1963 & $\mathbf{1 9 6 4}$ \\
\hline São Paulo & 18,0 & 18,0 & 19,0 & 15,0 \\
Manáus & - & 4,8 & 6,7 & 13,5 \\
Fortaleza & $\mathbf{5 5 , 5}$ & $\mathbf{8 8 , 2}$ & $\mathbf{9 7 , 9}$ & - \\
Salvador & - & $\mathbf{7 , 6}$ & $\mathbf{7 , 9}$ & $\mathbf{5 , 0}$ \\
Belo Horizonte & $\mathbf{9 , 6}$ & $\mathbf{9 , 9}$ & $\mathbf{1 4 , 0}$ & - \\
Rio de Janeiro & $\mathbf{9 , 3}$ & - & - & - \\
Curitiba & $\mathbf{1 3 , 6}$ & $\mathbf{1 5 , 2}$ & $\mathbf{2 0 , 9}$ & - \\
Pôrto Alegre & $\mathbf{1 3 , 4}$ & $\mathbf{1 5 , 0}$ & $\mathbf{2 0 , 8}$ & - \\
Goiânia & $\mathbf{1 0 , 5}$ & $\mathbf{9 , 3}$ & $\mathbf{7 , 2}$ & - \\
Niterói & $\mathbf{1 2 , 5}$ & $\mathbf{1 4 , 9}$ & $\mathbf{1 6 , 8}$ & - \\
Recife & - & 16,5 & $\mathbf{1 2 , 7}$ & 19,4 \\
& & & & \\
\hline
\end{tabular}

Fonte: Anuário Estatístico do Brasil (IBGE), 1965.
Quanto à freqüência do tipo de malformação, segundo aparelhos ou sistemas, predomina amplamente em todos os anos estudados, a mortalidade por malformações congênitas do aparelho cardio-circulatório. Esse aspecto será melhor analisado abaixo, na parte referente aos óbitos por malformaçóes em menores de $]$ ano.

\section{MORTALIDADE POR MALFORMACOES} CONGENITAS EM MENORES DE 1 ANO

Em relação à idade, a mortalidade por malformações congênitas ocorre em proporção acentuadamente maior em menores de 1 ano.

No período de 25 anos estudados, no Município de São Paulo, a média para todos os anos foi $81,3 \%$ dos óbitos por malformações, ocorrendo em menores de 1 ano (o mínino observado foi $77,8 \%$ em 1959 e o máximo, $85,8 \%$ em 1951).

$$
\text { T A B E L A } 3
$$

Malformações congênitas - Mortalidade (coef. $\times 100.000$ nascidos vivos) e mortalidade proporcional (\%) em menores de 1 ano - Munlcipio de são Paulo 1940-1964

\begin{tabular}{c|c|c}
\hline A n o & Coeficiente & $\%$ \\
\hline 1940 & 217,0 & 1,80 \\
1941 & 250,0 & 1,85 \\
1942 & 210,0 & 1,70 \\
1943 & 200,0 & 1,80 \\
1944 & 200,0 & 1,80 \\
1945 & 270,0 & 2,70 \\
1946 & 255,0 & 3,20 \\
1947 & 280,0 & 3,45 \\
1948 & 250,0 & 2,90 \\
1949 & 308,0 & 3,40 \\
1950 & 247,0 & 2,70 \\
1951 & 350,0 & 3,80 \\
1952 & 300,0 & 4,25 \\
1953 & 335,0 & 4,25 \\
1954 & 340,0 & 4,55 \\
1955 & 400,0 & 4,60 \\
1956 & 454,0 & 5,25 \\
1957 & 442,0 & 6,00 \\
1958 & 464,0 & 6,60 \\
1959 & 411,0 & 6,30 \\
1960 & 437,0 & 7,00 \\
1961 & 459,0 & 7,60 \\
1962 & 450,0 & $\mathbf{7 , 0 0}$ \\
1963 & 472,0 & 6,75 \\
1964 & 398,0 & 5,90 \\
& & \\
\hline
\end{tabular}


LAURENTI, R. - A mortalidade por malformação congênita no Município de São Paulo no periodo de 1940 a 1964 . Rev. Saúde públ., S. Paulo, 1(1):51-58, jun. 1967.

Na Tabela 3 estão os coeficientes de mortalidade infantil por malformaçōes congênitas de 1940-1964 e a tendência da mortalidade pode ser melhor analisada na Figura, onde se vê que embora a variação do coeficiente de ano para ano não seja constante e nem sempre no mesmo sentido, há entre os anos iniciais e finais do período uma nítida tendência ascendente da mortalidade.

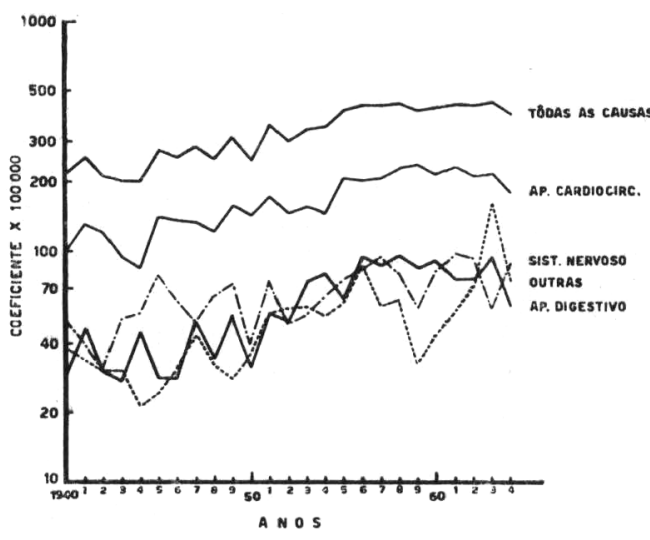

Figura - Mortalidade por malformações congênitas, em menores de 1 ano, no Município de São Paulo, 1940-1964. Coeficiente $\times 100.000$ nascidos vivos.

Para se medir a tendência ascendente da mortalidade por malformações congênitas em menores de 1 ano, analisaremos os coeficientes médios nos triênios 1940-42, 1950-52 e 1962-64, que são respectivamente: $225,0,299,0$ e $444,0 \times$ 100.000 nascidos vivos, onde se vê que o coefiicente médio de mortalidade infantil por malformações congênitas au- mentou do triênio 1940-42 para o triênio 1950-52 em 32,8\%; e do triênio 1950-52 para 1962-64 houve um aumento de $47,5 \%$. Relacionando-se os coeficientes médios inicial e final, houve um aumento de $95,5 \%$.

A mortalidade proporcional por malformações congênitas, em relação a todos os óbitos por outras causas, em menores de um ano, pode também ser avaliada, ano a ano, pela Tabela 3. A Tabela 4 mostra a mortalidade proporcional média por malformaçōes congênitas para os triênios 1940-42, 1950-52 e 1962-64, juntamente com 3 outros grupos de causas bastante importantes na mortalidade infantil no Município de São Paulo: doenças infecciosas e parasitárias, exceto sífilis, correspondendo aos números códigos 001-138 (exceto 020-029); doenças do aparelho respiratório correspondendo aos números códigos 480-483, 490-493 e $763,470-475$ e $500-527$; e lesôes devidas ao parto correspondendo aos números códigos 760.762 da Classificação Internacional de Doenças, Revisão de 1955.

Vemos que das 4 causas assinaladas, sòmente as moléstias infecciosas e parasitárias tiveram importância cada vez mais reduzida na porcentagem dos óbitos. Os outros 3 grupos apresentaram um aumento da mortalidade proporcio. nal, sendo, porém, que o aumento observado para as malformaçóes congênitas foi bem maior que para os outros grudos. Assim é que do $10^{\circ}$ para o $20^{\circ}$ triênio a mortalidade proporcional média aumentou de $100 \%$ para as malforma-

TABELA 4

Mortalidade proporcional média - Malformacões congênitas, doenpas do aparelho respiratório, moléstias infecciosas e parasitárias (exceto sifilis), lesões devidas ao parto - Triênios 1940-42, 1950-52 e 1962-64 - Município de São Paulo

\begin{tabular}{c|c|c|c|c} 
Triênios & $\begin{array}{c}\text { Malformacões } \\
\text { congênitas } \\
(\% \text { média) }\end{array}$ & $\begin{array}{c}\text { Infeccões } \\
\text { parasitártas } \\
(\% \text { média) }\end{array}$ & $\begin{array}{c}\text { Aparelho } \\
\text { respiratório } \\
\text { média) }\end{array}$ & $\begin{array}{c}\text { Lesões devidas } \\
\text { ao parto } \\
\text { (\% média) }\end{array}$ \\
\hline $1940-42$ & 1,8 & 10,6 & 16,4 & 3,7 \\
$1950-52$ & 3,6 & 5,4 & 21,7 & 7,0 \\
$1962-64$ & 6,5 & 4,8 & 26,9 & 11,7 \\
\hline
\end{tabular}


LAURENTI, R. - A mortalidade por malformação congênita no Município de São Paulo no período de 1940 a 1964. Rev. Saúde públ., S. Paulo, 1(1):51-58, jun. 1967.

ções congênitas, $30 \%$ para as moléstias do aparelho respiratório e $88 \%$ para as lesões devidas ao parto. Do $2 .^{\circ}$ para o $3 .^{\circ}$ triênio, a média foi aumentada $124 \%$ para as malformações congênitas, $23 \%$ para as doenças do aparelho respiratório e $67 \%$ para as lesões devidas ao parto.

0 coeficiente de mortalidade infantil por malformações congênitas, segundo o sexo, foi maior para o sexo masculino em todos os anos estudados. Assim tivemos, por exemplo, para 1963 um coeficiente para o sexo masculino de $520 \times$ 100.000 nascidos vivos masculinos e $421 \times 100.000$ nascidos vivos femininos. Esses coeficientes podem ser comparados aos de muitos países, onde tambem foi verificado maiores coeficientes para o sexo masculino (HigGins ${ }^{2}, 1965$ ).

Analisando os óbitos ocorridos por malformações congênitas em menores de 1 ano, vê-se que a maioria ocorre antes do $10^{\circ}$ mês de vida. Na Tabela 5 estão expostas as percentagens dos óbitos que ocorrem antes do $1 .^{\circ}$ mês de vida, em relação aos ocorridos em menores de 1 ano, e os coeficientes de mortalidade neonatal por malformações congênitas, desde 1950 a 1964, que foram os anos para os quais foi possível a obtenção dos dados.

A mortalidade por malformações congênitas, em menores de 1 ano, segundo o tipo de malformação evidenciou uma nítida predominância das malformações do aparelho cárdio-circulatório. Isto também ocorre em outros países (HIGGiNs ${ }^{2}$, 1965).

De 1940 a 1964 a mortalidade por malformações do aparelho cárdio-circulatório representou, em média, $48,5 \%$ de tôdas as malformações, variando de um mínimo de $40,71 \%$ em 1944 até um máximo de 57,9\% em 1959. A seguir vêm as malformaçôes do sistema nervoso, correspondendo a uma média de $20,1, \%$ (máximo de 26\% e um mínimo de $13,7 \%$ em 1954).
T A B E L A 5

Mortalidade néo-natal por malformaçôes congênitas (coeficiente $\times 100.000$ nascidos vivos) - Proporção dos óbitos por malformaçóes congênitas em menores de 1 ano, ocorridos antes de 1 més de vida (\%) - Municipio de São Paulo, 1950-1964)

\begin{tabular}{l|c|c}
\hline Anos & Coeficiente & $\%$ \\
\hline 1950 & 136 & $\mathbf{5 4 , 9}$ \\
1951 & 217 & 62,3 \\
1952 & 193 & $\mathbf{5 1 , 5}$ \\
1953 & 172 & 60,0 \\
1954 & 176 & 52,0 \\
1955 & 222 & 55,8 \\
1956 & 249 & $\mathbf{5 4 , 8}$ \\
1957 & 246 & $\mathbf{5 5 , 7}$ \\
1958 & 260 & $\mathbf{5 5 , 9}$ \\
1959 & 225 & $\mathbf{5 4 , 7}$ \\
1960 & 242 & $\mathbf{5 5 , 3}$ \\
1961 & 245 & 53,4 \\
1962 & 281 & $\mathbf{5 7 , 8}$ \\
1963 & 216 & 45,8 \\
1964 & 249 & $\mathbf{6 3 , 6}$ \\
\hline
\end{tabular}

As malformações do aparelho digestivo aparecem em terceiro lugar com uma média de $17 \%$ (máximo 20,6\% em 1958 e mínimo de 10,7\% em 1945).

Vemos que as malformaçōes dos sistemas cárdio-circulatório, digestivo e nervoso, juntamente, são responsáveis por mais de $90 \%$ da mortalidade por malformações em menores de 1 ano, ficando o restante para tôdas as outras causas: geniturinário, fissura palatina e lábio leporino, ossos e articulaçōes, malformaçōes não especificadas e outras. As malformações do aparelho cárdio-circulatório são aquelas que correspondem ao número código 754 da Classificação Internacional de Doenças, Revisão de 1955; as do sistema nervoso correspondem aos números códigos 750-753; do aparelho digestivo, ao número 756 ; as fissuras do palato e lábio leporino correspondem ao número 755 ; as malformações do aparelho geniturinário ao número 757 ; as mal. formações dos ossos e das articulações correspondem ao número 758. O número 759 inclui os outros vícios de malformações congênitas e as não especificadas. 
LAURENTI, R. - A mortalidade por malformação congênita no Municíplo de São Paulo no periodo de 1940 a 1964. Rev. Saúde públ., S. Paulo, 1(1):51-58, jun. 1967.

T A BE L A 6

Mortalidade segundo os tipos de malformaçóes congênitas (coeficiente $\times 100.000$ nascidos vivos) - Município de São Paulo - Anos 1940-1964

\begin{tabular}{|c|c|c|c|c|c|c|c|}
\hline Anos & $\begin{array}{l}\text { Todos } \\
\text { os tipos }\end{array}$ & $\begin{array}{l}\text { Sistema } \\
\text { nervoso }\end{array}$ & $\begin{array}{l}\text { Cardiocir- } \\
\text { culatório }\end{array}$ & $\begin{array}{l}\text { Fiss. pal. e } \\
\text { láb. lepor. }\end{array}$ & Digest. & $\begin{array}{l}\text { Genitu- } \\
\text { rinário }\end{array}$ & Outros \\
\hline 1940 & 217 & 50 & 100 & 12 & 29 & 3 & 23 \\
\hline 1941 & 250 & 40 & 130 & 6 & 46 & 8 & 20 \\
\hline 1942 & 210 & 30 & 120 & 13 & 30 & 0 & 17 \\
\hline 1943 & 200 & 50 & 93 & 16 & 27 & 5 & 9 \\
\hline 1944 & 200 & 53 & 83 & 7 & 43 & 2 & 12 \\
\hline 1945 & 270 & 78 & 140 & 12 & 28 & 5 & 7 \\
\hline 1946 & 255 & 61 & 137 & 6 & 28 & 4 & 19 \\
\hline 1947 & 280 & 50 & 134 & 10 & 50 & 8 & 28 \\
\hline 1948 & 250 & 63 & 121 & 12 & 34 & 7 & 13 \\
\hline 1949 & 308 & 72 & 157 & 8 & 53 & 3 & 15 \\
\hline 1950 & 247 & 39 & 142 & 1 & 31 & 3 & 31 \\
\hline 1951 & 350 & 74 & 171 & 7 & 54 & 7 & 37 \\
\hline 1952 & 300 & 48 & 147 & 23 & 49 & 9 & 24 \\
\hline 1953 & 335 & 52 & 153 & 15 & 73 & 7 & 35 \\
\hline 1954 & $\mathbf{3 4 0}$ & 63 & 146 & 14 & 79 & 6 & 32 \\
\hline 1955 & 400 & 75 & 204 & 17 & 62 & 3 & 39 \\
\hline 1956 & 454 & 83 & 203 & 18 & 94 & 4 & 52 \\
\hline 1957 & 442 & 94 & 204 & 14 & 86 & 5 & 39 \\
\hline 1958 & 464 & 80 & 228 & 15 & 95 & 8 & 38 \\
\hline 1959 & 411 & 56 & 238 & 12 & 84 & 6 & 15 \\
\hline 1960 & 437 & 82 & 218 & 11 & 90 & 5 & 31 \\
\hline 1961 & 459 & 97 & 230 & 6 & 75 & 7 & 44 \\
\hline 1962 & 450 & 93 & 210 & 21 & 76 & 5 & 45 \\
\hline 1963 & 472 & 58 & 213 & 13 & 95 & 11 & 82 \\
\hline 1964 & 398 & 86 & 180 & 11 & 58 & 8 & 55 \\
\hline
\end{tabular}

Na Tabela 6 estão expostos os coeficientes $(\times 100.000$ nascidos vivos $)$ para os diferentes tipos de malformações congênitas. Nessa tabela, as "outras" malformações incluem: ossos, articulações, não especificados e outros.

$\mathrm{Na}$ Figura, além da mortalidade infantil por malformações congênitas, está também evidenciada a tendência da mortalidade pelos 3 tipos predominantes de malformações (cárdio-ciculatórias, nervosas e digestivas). A análise da Tabela 6 e da Figura mostram que os coeficientes de mortalidade por aparelhos variam de ano a ano, mostrando porém no geral uma tendência ascendente. A tendência ascendente da mortalidade por malformações do aparelho digestivo e do sistema nervoso é bastante irregular, havendo de ano a ano variações bastante acentuadas ora para mais, ora para me- nos. Embora no geral predomine os coeficientes de mortalidade por malformações do sistema nervoso, há em alguns anos $(1942,1952,1953,1954,1956$, $1958,1959,1960,1963)$ coeficientes maiores para as malformações do aparelho digestivo, em relação às do sistema nervoso.

Ainda que também para as malformações do aparelho cárdio-circulatório a mortalidade evolui com variações nem sempre constantes e nem sempre no mesmo sentido, a tendência ascendente é mais uniforme do que para os demais tipos de malformações.

\section{DIS CUSSAO}

Os dados apresentados mostram que a mortalidade por malformações congênitas no Município de São Paulo no período compreendido entre 1940 e 1964, aumen- 
LAURENTI, R. - A mortalidade por malformação congênita no Município de São Paulo no periodo de 1940 a 1964. Rev. Saúde públ., S. Paulo, 1(1):51-58, jun. 1967.

tou. Para justificar êsse aumento algumas hipóteses poderiam ser aventadas como: o aprimoramento do diagnóstico das malformações congênitas; a vinda de doentes de outras áreas em busca de tratamento em São Paulo e também um aumento na incidência das malformações congênitas.

0 aprimoramento da qualidade diag. nóstica seria - a nosso ver - um importante fator responsável pelo aumento da mortalidade. Apareceriam atualmente casos que antes não eram diagnosticados e essa melhora do diagnóstico estaria condicionada pelo avanço que tem havido na terapêutica clínico-cirúrgica das malformações. Juntamente com a melhora do diagnóstico um outro fator bastante importante seria aquêle dado por pacientes que procuram São Paulo em busca de tratamento médico, aqui falecendo e elevando artificialmente a mortalidade por malformações congênitas no Município. Esse fator já foi bem estudado e representa, realmente, um contingente importante, os óbitos de indivíduos procedentes de outras área (BARBOSA ${ }^{1}, 1967$ ).

Esse último fator, ou seja, a vinda de pacientes de outras áreas para São Paulo, poderia de certa forma ser excluído ou diminuído ao máximo se fôssem estudados os coeficientes de mortalidade por malformações congênitas em crianças falecidas nos primeiros sete dias de vida. Isso admitindo-se - o que parece ocorrer - que com essa idade é nula ou mínima a vinda de pacientes de outras áreas para São Paulo, considerando-se que a gravidade da malformação já se manifesta nos primeiros momentos após o nascimento, impedindo ou dificultando então o deslocamento para a capital.

Calculando-se os coeficientes de mortalidade por malformações congênitas para menores de 7 dias, a partir do ano que se obteve êsses dados, ou seja, de 1950 a 1964 vê-se que há também uma tendência ascendente. Os coeficientes médios para o triênio $1950-52$ foi $125,26 \times$ 100.000 nascidos vivos, e para o triênio $1962-64$ foi $161,91 \times 100.000$ nascidos vivos. Houve, portanto, um aumento de $29,26 \%$. Poder-se-ia então dizer que provàvelmente êsse aumento não teria sofrido a influência da vinda de doentes de fora e aqui falecidos, mas sim que seria devido à melhora havida no diagnóstico ou mesmo poder-se-ia supor estar havendo um aumento real da incidência de malformações congênitas. Esse é um aspecto bastante interessante para investigações futuras.

\section{CONCLUSOES}

1 - A mortalidade por malformações congênitas, no município de São Paulo, no período de 1940 a 1964 está apresentando uma tendência ascendente.

2 - De cada 100 óbitos por malformações congênitas, aproximadamente 80 ocorrem em menores de 1 ano. Entre os menores de 1 ano, $50 \%$ ocorre abaixo de 1 mês de vida.

3 - E maior a mortalidade por malformações congênitas no sexo masculino.

4 - Segundo os aparelhos ou sistemas afetados, predominou amplamente em todos os anos a mortalidade por malformações do aparelho cárdio-circulatório (aproximadamente $50 \%$ de tôdas as malformações). A seguir, em ordem decrescente, situam-se as malformações do sistema nervoso, as do aparelho digestivo, as fissuras do palato e lábio leporino, do aparelho geniturinário e outros (incluindo ossos, articulações, malformações não especificadas e outras).

\section{S U M M A R Y}

The mortality by congenital malformation has been studied in the county of São Paulo, during a 25 - year period from 1940 to 1964 . The observed trend is ascending and predominant under one year old, as well as in male in relation to female. The most frequent kinds of letal congenital malformations were from the cardiovascular system. The most probable causes of the increasing mortality rates from congenital malformations are discussed. 
LAURENTI, R. - A mortalidade por malformação congênita no Município de são Paulo no periodo de 1940 a 1964. Rev. Saúde públ., S. Paulo, 1(1):51-58, Jun. 1967.

\section{REFERENCIAS BIBLIOGRAFICAS}

1. BARBOSA, V. - Estudo comparativo entre os óbitos "de fato" $e$ "de direito" no Municipio de São Paulo. São Paulo, 1967. 86 p. (Tese de docência livre - Fac. Hig. Saúde Pública da USP).

2. HIGGINS, I. T. T. - The epidemiology of congenital heart disease. $J$. Chron. Dis., 18(8) :669-721, Ag. 1965.

3. MALPAS, P. - The incidence of human malformations and the significance of changes in the maternal environment in their causation. J. Obstet. Gynaec. Brit. Emp. 44(3):434-454, June 1937.

4. McINTOSH, $R$. et alil. - The incidence of congenital malformations: a study of 5.964 pregnancies. Pediatrics, 14(5):505522, Nov. 1954.

5. SALDANHA, P. F. et alli. - Incidêncla de defeitos congênitos na população de São Paulo. Rev. Paul. Med. 63(4): 211-229, out. 1963.

6. SHAPIRO, R. N. et alli. - The incidence of congenital anomalies discorvered in the neonatal period. Amer. $J$. Surg. $96(3): 396-400$, sept. 1958. 